\title{
A Simple Electronic Circuit for an Automatic Train Safety Stop System
}

\author{
Andre Donza \\ Department of Electrical Engineering \\ Federal University of Para \\ Department of Trains Maintenance, \\ Brazilian Company of Urban Trains \\ (CBTU), Recife, Brazil \\ andredonza@cbtu.gov.br
}

\author{
Riley Jones \\ Laboratory of Electronics \\ Brazilian Company of \\ Urban Trains (CBTU), \\ Recife, Brazil \\ rileyrupert@cbtu.gov.br
}

\author{
Rafael Teixeira \\ Department of Trains \\ Maintenance \\ Brazilian Company of Urban \\ Trains (CBTU), \\ Recife, Brazil \\ rafaelteixeira@cbtu.gov.br
}

\author{
Bruno da Silva \\ Department of Trains \\ Maintenance \\ Brazilian Company of \\ Urban Trains (CBTU), \\ Recife, Brazil \\ brunogsilva@cbtu.gov.br
}

\begin{abstract}
A particular safety system known as "dead man's circuit" detects the presence of human command in the conduction of the trains operated by the Brazilian Company of Urban Trains (CBTU) in Recife, Brazil, thus consisting a security mechanism that makes automatic braking of any train composition circulating in the transportation system of the city feasible in incidents of loss or apparent loss of driving. Originally, the electrical signal indicating human control in the conduction of the vehicles came from the descent of the traction lever in the driving cabins, which caused the switching of a pair of electrical contacts. Given the importance of the system and the need to avoid repetitive strain injuries to drivers, the need to push the traction knob was eliminated by the use of an electronic device developed by an outsourced company and deployed in the trains of the older fleet. However, the high failure and downtime rates associated with the circuit acquired from the referred contracted company caused the need to develop a more robust and maintenance-friendly design for the dead man's system. In fact, despite the good performance of the purchased system, the strength of the resin coating that accommodated the capacitive touch sensor assembly at the end of the traction lever prevented the corresponding electronic circuit from any access for research or corrective interventions. Thus, the content of this paper essentially presents the specifications and the description of operation of the alternative electronic circuit designed for the dead man's system, which is operating normally without electrical defects in $\mathbf{4}$ old trains for almost a year.
\end{abstract}

Keywords-urban trains; safety system; touch sensor; automatic braking

\section{INTRODUCTION}

A large number of techniques based on different maintenance approaches have been proposed to solve various types of maintenance problems [1]. These techniques and approaches must be adequate to specific situations and must be adapted to the singular needs of a company [2]. Obviously, in any approach, reliability assessments play a key role in determining whether or not a critical system or equipment should be replaced. The older fleet of the metropolitan trains operated by the Brazilian Company of Urban Trains (CBTU) in Recife, Brazil, is composed by vehicles from a Brazilian company, namely CISM. A particular system of these trains, known as dead man's circuit detects the presence of human command in the conduction of the trains, thus consisting of a security mechanism that makes feasible in incidents of loss or apparent loss of driving, automatic braking of any train composition circulating in the transportation system of the city. The high failure and downtime rates associated to the dead man system developed by an outsourced company for the old fleet caused the need to deploy another design of this system. Thus, the current paper essentially presents the specifications and the description of operation of the alternative electronic circuit designed for the dead man's system, which is operating normally without electrical defects in 4 trains since August 2017.

\section{HISTORY OF THE SYSTEM}

Originally, the electrical signal indicating human control in the conduction of the old railway vehicles of CISM came from the descent of the traction lever in the driving cabins, which caused the switching of a pair of electrical contacts, being continuously necessary for the driver to be with a hand supported on the traction knob to get or keep any train moving. Given the importance of the system and the need to avoid repetitive strain injuries to drivers, the need to push the traction lever to conduct the trains of the old fleet was eliminated by the use of a set consisting of two devices: a touch sensor, installed at the end of the traction handles, and an electronic main module, both shown in Figure 1, developed and installed by an outsourced company admitted by CBTU. Despite the ergonomic advantage, the implemented modification did not increase the reliability of the system and the maintenance quality. The strength of the resin coating that accommodated the touch sensor assembly at the end of the traction lever prevented the corresponding electronic circuit from any access for research or corrective interventions. Due to the lack of technical documentation of the circuits implemented by the outsourced company and the impossibility of accessing the sensor assembly without damaging it, the initial solution adopted to reduce the time of unavailability of trains for commercial operation due to failures in the dead man system was the acquisition of adjustable sensitivity capacitive 
cylindrical sensors, which were installed at the end of the traction levers on leaked silicone beads (Figure 2), produced by the CBTU's own workshop, replacing the previously adopted resin encapsulated assembly. Despite the effectiveness and simplicity of the new device, the routing of the electrical cables of the capacitive sensor by very tight ducts, a condition aggravated by the vibrations of the trains in operation, and possible inversions of the electrical connections between the main control module and the sensor, caused short circuits and reverse polarizations difficult to avoid due to the extreme limitations of space that made it difficult to install any simple electrical or mechanical protection device.
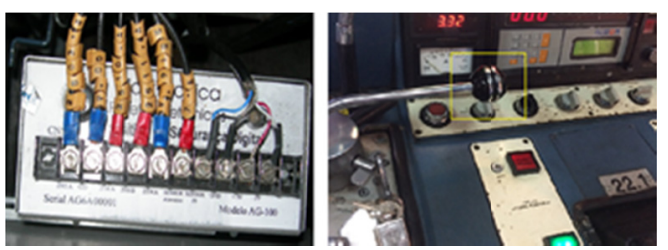

Fig. 1. Main module box in the left and cover of the old capacitive touch sensor in the right

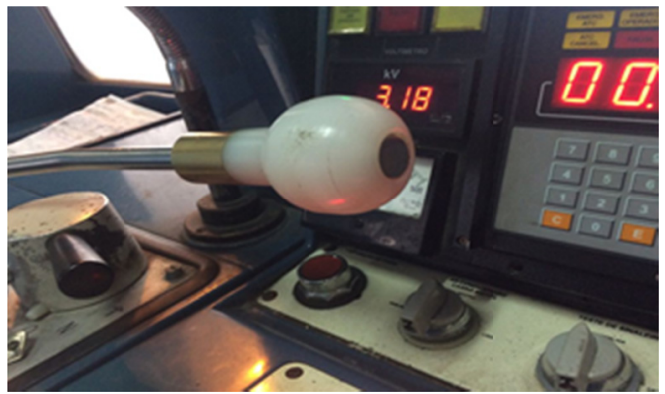

Fig. 2. First change in the traction lever. Capacitive sensors were installed on leaked silicone beads.

Therefore, given the particular conditions of the trains of the old fleet, the capacitive sensor, despite its known reliability, together with the main control module developed by the outsourced company, did not seem to be the best option for the dead man's system. The high cost and the relative fragility experienced in the old trains raised the need of a new reliable and cost-effective touch sensor along with a compatible control module, in order to adjust the operation of such security system in the trains of the old fleet. In general, however, proximity capacitive sensors are rapidly proliferating across thousands of consumers, industrial and automotive applications [3], with a wide range of advantages. The major disadvantages of capacitive sensors are susceptibility to electromagnetic noise, sensitivity to temperature, non-linear response and hysteresis [4]. As shown in Figure 3, in the first half of 2017, 14\% of the failures of the dead man's system came from the main control module, $6 \%$ from electrical cables or connections between the traction lever and the control module and $29 \%$ were originated in the capacitive sensor of the traction lever or in the contacts of the old electromechanical system. From these $29 \%$ of failures directly related to the traction lever, $80 \%$ came from the capacitive sensors and $20 \%$ from the contacts of the old electromechanical system, as presented in Figure 4. The remaining $51 \%$ is almost entirely caused by equipment failures from the extensive brake circuit of the trains, which receives the electrical signal from the main control module of the dead man's system for processing. Thus, this percentage refers to a large set of equipment formed by solid state relays, programmable linear controllers, electric cables, bypass keys and electromechanical relays of the general brake control system.

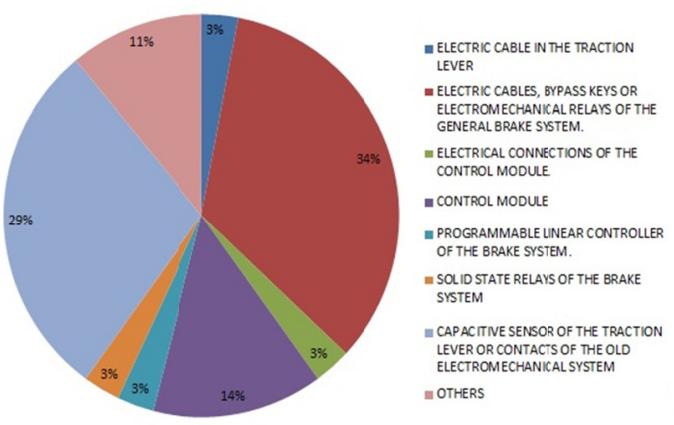

Fig. 3. Percentage of equipment failures during the first half of 2017 in the dead man's system.

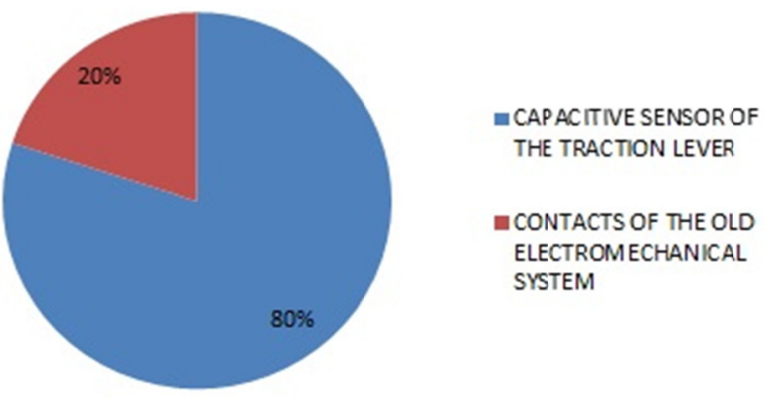

Fig. 4. Percentage of equipment failures directly related to the traction lever in the first half of 2017

\section{DESCRIPTION OF THE GENERAL ASSEMBLY}

IC 555 is the main component of the new touch sensor placed at the end of the traction lever to replace the capacitive sensors, as shown in Figures 5 and 6 . Three cables connect the sensor to the main module, powered by the $24 \mathrm{~V}$ DC voltage source of the radio system in the train. The first one starts from the output of the voltage regulator (L7812), taking $12 \mathrm{~V}$ DC to pin 8 of the " 555 ". The second connects the base of the transistor Q1 to the output pin 3 of the IC. The last of them connects to the ground, providing negative reference to pin 1 . The main components of the touch sensor are:

- IC 555

- Diode 1N4004 (installed behind the board, so not seen in Figure 5).

- $15 \mathrm{MOhms}$ resistor of $1 / 8 \mathrm{~W}$ (or two resistors in series with total resistance close to that)

- Electrolytic capacitor of $220 \mathrm{uF} / 16 \mathrm{~V}$. 
- $100 \mathrm{nF}$ polyester capacitor.

- $100 \mathrm{kOhms}$ resistor.

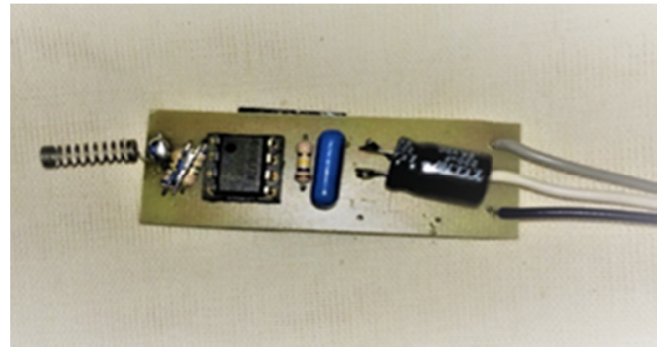

Fig. 5. New touch sensor with IC 555

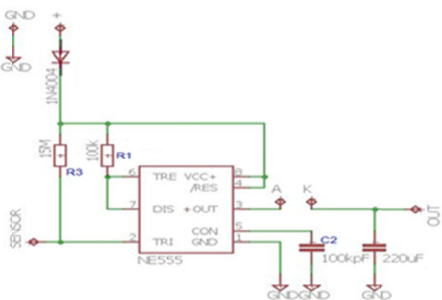

Fig. 6. Electrical circuit diagram of the touch sensor

As pointed out in [5], the main reasons of using the IC 555 are its stability, cheap cost and easy availability. The sensing board (Figure 7), measures $55 \mathrm{~mm} \times 29 \mathrm{~mm}$, while the control module board, shown in Figure 8 , is $100 \mathrm{~mm} \times 100 \mathrm{~mm}$. The main components of the control module board are:

- 2 relays of $12 \mathrm{~V} / 7 \mathrm{~A}$.

- IC 7812 (Voltage regulator).

- Electrolytic capacitor of $1 \mathrm{uF} / 50 \mathrm{~V}$.

- $7,5 \mathrm{kOhms}$ resistor of $1 / 8 \mathrm{~W}$.

- $1 \mathrm{kOhm}$ resistor of $1 / 8 \mathrm{~W}$.

- Transistor Bc546b.

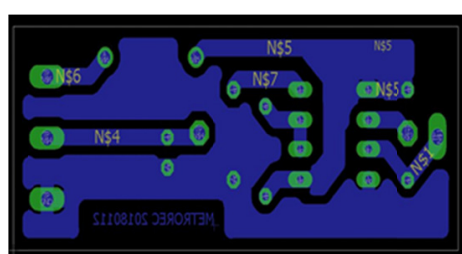

Fig. 7. Design of the sensing board

NE555 timer IC is an 8 pin dip package IC with a comparator, a voltage divider and a SR flip-flop, with the following pins $[6,7]$ :

- Trigger: For voltages below $1 / 3$ of the voltage source on this pin, the output is high.

- Threshold: Connected to the first comparator at the noninverting input terminal.
- Reset: Turns the output of the IC 555 to zero, independently of the trigger input.

- Control: By imposing a voltage on this pin or by connecting a pot between this pin and the ground, the pulse width of the output wave can be varied. When not used, the control pin should be bypassed to ground with a $0.01 \mathrm{uF}$ capacitor to prevent any noise problem.

- Discharge: Used to discharge the timing capacitor in astable and monostable modes.

- Output: Loads are tied to this pin.

- Ground: Reference point connected to the ground terminal of the DC voltage source.

- Supply: The power supply can vary from $5 \mathrm{~V}$ to $18 \mathrm{~V}$.

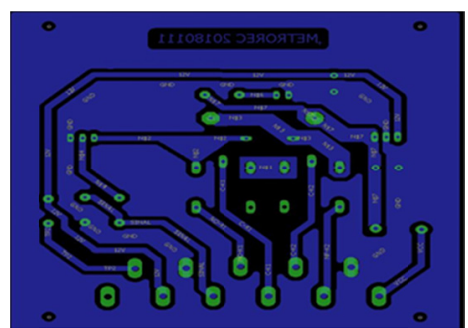

Fig. 8. Design of the main control module board

\section{OPERATING MODE}

The voltage regulator $\mathrm{L} 7812$ (U3) receives $24 \mathrm{~V}$ DC from the voltage source of the radio system in the train. The IC 555, in turn, is powered by the GND (1) and VCC (8) pins, receiving $12 \mathrm{~V}$ DC from the voltage regulator output, which is filtered by the $100 \mathrm{nF}$ capacitor $\mathrm{C} 4$ to avoid electrical oscillations, as shown in Figures 9 and 10.

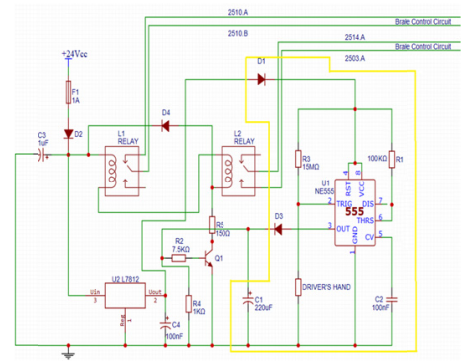

Fig. 9. Simplified electrical schematic diagram of the touch sensor (highlighted in yellow) along with the control module of the dead man's system

The use of the control pin of the IC 555 is unnecessary, so the capacitor $\mathrm{C} 2$ was placed between terminals 1 and 5 to prevent voltage fluctuations from affecting the sensitivity of the integrated circuit. In the voltage input circuit, the fuse F1 cuts currents greater than $1 \mathrm{~A}$, the capacitor C3 filters the voltage delivered by the radio system and the LED D2 indicates the activation of the control module. The driver's hand acts as a touch switch on the 555's trigger terminal (pin 3). To refine the contact and ensure good performance, metal receivers have 
been incorporated into the sensor circuit board, as shown in Figure 11.

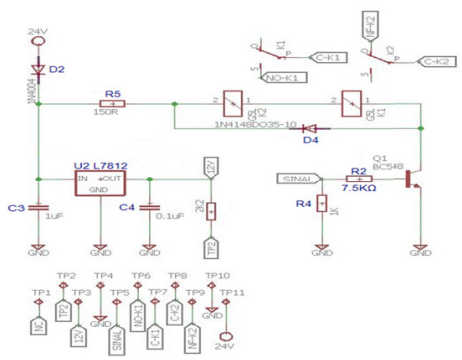

Fig. 10. Electrical circuit diagram of the control module

As the 555 trigger is activated for voltages below $1 / 3$ of the magnitude of the supply voltage, its output state switches to high level when one of the hands of the driver touches the receiver, which causes, as already seen, the energization of the relays $\mathrm{K} 1$ and $\mathrm{K} 2$. The input impedance of the 555 trigger (terminal 2) is quite high and, without touching the metallic receiver, the level is high $(\mathrm{Vcc})$ on that port. When hand contact occurs, a potential difference is established by the induced voltage in the human body, turning the input of port 2 to low level. Then the switching of relays K1 and K2 happens rapidly, only subjected to the rapid loading or unloading of the capacitor $\mathrm{C} 1$. The operation of the sensor inside a train is slightly different from its behavior outside. Since the train is a closed metal structure, a simple touch on the handle is not enough to activate the 555's output. Therefore, the copper ring of the traction handle was extended, to enable the passage of a very small electric current by the operator's hands (Figure 12).

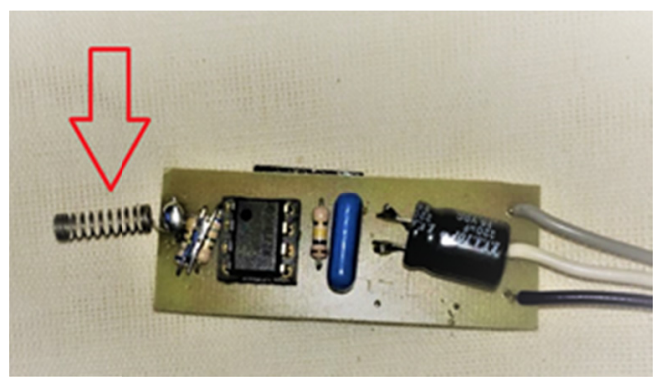

Fig. 11. Metallic receptor for trigger terminal

The transistor Q1, responsible for switching the state of the contacts of the relays $\mathrm{K} 1$ and $\mathrm{K} 2$, must operate in the saturation region. Considering the minimum transistor current gain equal to 110 , as reported in the corresponding datasheet, and the collector saturation current of approximately $160 \mathrm{~mA}$, as shown in (1), the base current must be at least $1.45 \mathrm{~mA}$ for the transistor to operate as a closed switch, as detailed in (2).

$$
\begin{aligned}
& I_{C(\mathrm{SAT})}=\frac{V_{\mathrm{CC}}}{R_{5}}=\frac{24}{150}=160 \mathrm{~mA} \\
& I_{B_{\mathrm{MIN}}(\mathrm{SAT})}=\frac{160}{110}=1,45 \mathrm{~mA}
\end{aligned}
$$

The resistor $\mathrm{R} 2$ of 7,5k $\Omega$ then ensures satisfactorily the operation of the transistor in the saturation region, since the base current produced by the output of the IC 555 is $1,51 \mathrm{~mA}$, as described in (3).

$$
I_{B}=\frac{V_{\mathrm{BB}}-V_{\mathrm{BE}}}{R_{2}}=\frac{12-0,7}{7,5 k}=1,51 \mathrm{~mA}>1,45 \mathrm{~mA}
$$

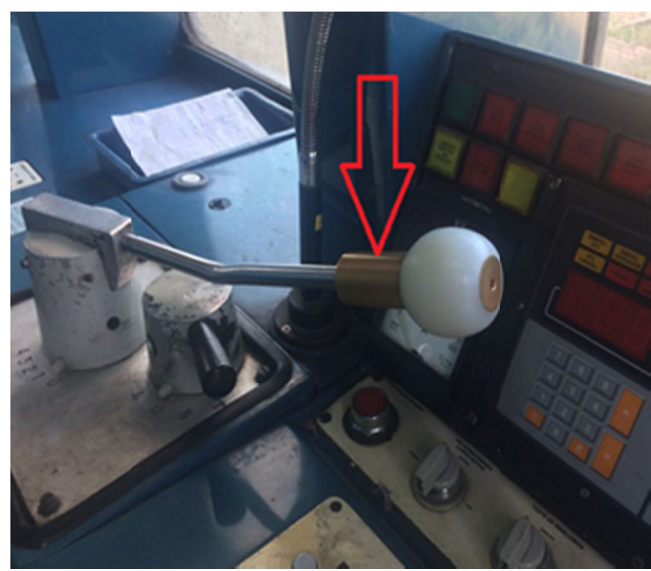

Fig. 12. Traction lever with extended metal ring and with the new touch sensor installed.

Resistor R4 limits the discharge time of capacitor $\mathrm{C} 1$ to a maximum of $0.48 \mathrm{~s}$ as shown in (4).

$$
\begin{aligned}
& t_{\text {discharge }}=-\left(R_{2} \| R_{4}\right) * C * \ln \frac{V_{C}(t)}{V_{0}}=\ldots \\
& \ldots=-(7,5 k \| 1 k) * 220 * 10^{-6 *} \ln \frac{0,7}{12}=0,48 s
\end{aligned}
$$

Then, after almost half a second, the voltage of the common point between resistors $\mathrm{R} 2$ and $\mathrm{R} 4$ drops to about $0.7 \mathrm{~V}$. Therefore, when the driver pulls his hands off the traction lever, making terminal 3 of the IC 555 receive a high voltage again, the transistor takes less than half a second to operate as an open switch and to deactivate relays $\mathrm{K} 1$ and $\mathrm{K} 2$. The system is protected against reverse voltage by the diode D4 in such a way that if at the moment of suppression of current there is induction of high current in the coils of the relays, the diode, being polarized in the same direction of the induced voltage, will absorb the energy that could damage the transistor Q1.

\section{INSTALLATION}

The mounting process is simple and takes about 1 hour. In the main module, shown in Figure 13, the connections follow the scheme outlined in Table I.

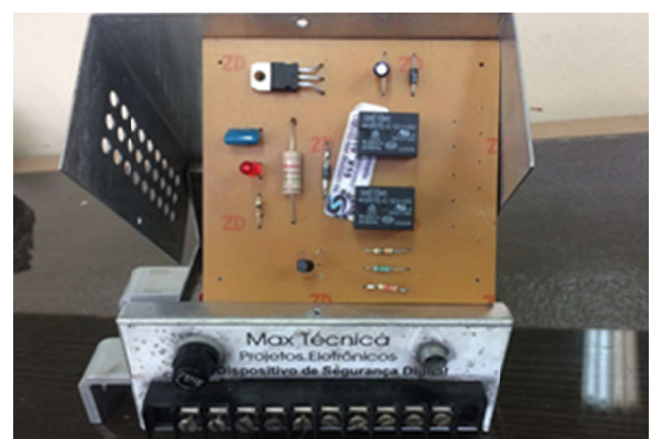

Fig. 13. Main module enclosure and electronic board 
TABLE I. ELECTRICAL CONNECTIONS IN THE MAIN MODULE BOX

\begin{tabular}{|c|c|c|}
\hline \multirow{2}{*}{ Terminal } & \multicolumn{2}{|c|}{ Electrical Connections } \\
\cline { 2 - 3 } & Cable & Function \\
\hline $7 \mathrm{~A}$ & $7 . \mathrm{A}$ & $24 \mathrm{Vcc}$ \\
\hline GD & GD.A & GD \\
\hline $2510 . \mathrm{A}$ & $2510 . \mathrm{A}$ & NC Contact \\
\hline $2510 . \mathrm{B}$ & $2511 . \mathrm{A}$ & NC Contact \\
\hline $2514 . \mathrm{A}$ & $2514 . \mathrm{A}$ & NO Contact \\
\hline $2503 \mathrm{~A}$ & $2503 . \mathrm{A}$ & NO Contact \\
\hline 3 & White & Signal \\
\hline 2 & Grey & GD \\
\hline 1 & Black & $12 \mathrm{Vcc}$ \\
\hline
\end{tabular}

a. Scheme of connections in the main module board.

\section{CONCLUSION}

The decision about replacing a critical system is sometimes controversial within the planning of the maintenance processes of a company. In fact, resolutions such as these consume time, resources, manpower, and should be carried out by objective considerations about the costs and benefits of the change. Therefore, its effects should also be revised so that new actions can be taken after the disclosure of any unwelcome unrolling. So far, no incident has exposed any relevant vulnerability of the new dead man's system, which is operating normally in four trains for almost a year. There are no electronic components damaged in these vehicles and no complaints from drivers regarding the new traction levers. Although no unexpected event has happened, the operation of the new system continues to be monitored to evaluate the durability and the reliability of the system. In the path between the dead man's system and the programmable logic controller of the brake control circuit, there is a device that can have its timing function incorporated through the CI 555 of the touch sensor. Future developments of the project include this modification.

\section{REFERENCES}

[1] R. Ahmad, S. Kamaruddin, "Application and Comparison of Three Maintenance Techniques for Replacement Decision Making - a Case Study in the Pulp Manufacturing Industry", International Journal of Process Systems Engineering, Vol. 2, No. 2, pp. 135-153, 2012

[2] E. Viles, D. Puente, M. J. Alvarez, F. Alonso, "Improving the corrective maintenance of an electronic system for trains", Journal of Quality in Maintenance Engineering, Vol. 13, No. 1, pp.75-87, 2007

[3] B. Osoinach, Proximity capacitive sensor technology for touch sensing applications, White Paper, FreeScale Semiconductor, 2008

[4] Z. Kappassov, J. A. Corrales, V. Perdereau, "Tactile sensing in dexterous robot hands - Review", Robotics and Autonomous Systems, Vol. 74, Part A, pp. 195-220, 2015

[5] V. Gupta, A. Deb, "Speed Control of Brushed DC Motor for Low Cost Electric Cars", IEEE International Electric Vehicle Conference, Greenville, USA, March 4-8, 2012

[6] K. Kotcherlakota, V. Chinta, "Smart Home Automation Based on 555 Timer", International Journal of Engineering Research and Application, Vol. 6, No. 5, pp. 77-80, 2016

[7] H. Goyal, "Understanding of IC 555 Timer and IC 555 Timer Tester", International Journal of Inventive Engineering and Sciences, Vol. 3, No. 2, pp. 4-6, 2015 